\title{
Remarks on the Phaseless Inverse Uniqueness of a Three-Dimensional Schrödinger Scattering Problem
}

\author{
Lung-Hui Chen \\ Department of Mathematics, National Chung Cheng University, 168 University Rd., Min-Hsiung, Chia-Yi County 621, Taiwan
}

Correspondence should be addressed to Lung-Hui Chen; mr.lunghuichen@gmail.com

Received 31 August 2016; Accepted 19 October 2016

Academic Editor: Ricardo Weder

Copyright ( 2016 Lung-Hui Chen. This is an open access article distributed under the Creative Commons Attribution License, which permits unrestricted use, distribution, and reproduction in any medium, provided the original work is properly cited.

We consider the inverse scattering theory of the Schrödinger equation. The inverse problem is to identify the potential scatterer by the scattered waves measured in the far-fields. In some micro/nanostructures, it is impractical to measure the phase information of the scattered wave field emitted from the source. We study the asymptotic behavior of the scattering amplitudes/intensity from the linearization theory of the scattered wave fields. The inverse uniqueness of the scattered waves is reduced to the inverse uniqueness of the analytic function. We deduce the uniqueness of the Schrödinger potential via the identity theorems in complex analysis.

\section{Introduction}

In this paper, we study the scattering problem of the Schrödinger equation given by

$$
\begin{aligned}
& \Delta_{x} u(x, k, y)+k^{2} u(x, k, y)-q(x) u(x, k, y) \\
& =-\delta(x-y), \quad x \in \mathbb{R}^{3}, \Im k \leq 0 ; \\
& u(x, k, y)=u_{0}(x, k, y)+u_{s}(x, k, y) ; \\
& \lim _{r \rightarrow \infty} r\left\{\frac{\partial u(x, k, y)}{\partial r}-i k u(x, k, y)\right\}=0,
\end{aligned}
$$

in which the nonzero $q(x)$ is the Schrödinger potential, $y \in$ $\mathbb{R}^{3}$ is the source point, $r:=|x-y|$, and

$$
u_{0}(x, k, y):=\frac{\exp \{-i k|x-y|\}}{4 \pi|x-y|}
$$

The perturbation is defined inside

$$
\Omega:=\left\{x \in \mathbb{R}^{3}|| x \mid<B, B>0\right\}
$$

with boundary $S$. In this paper, we assume

$$
\begin{aligned}
& q(x) \in \mathscr{C}^{4}\left(\mathbb{R}^{3} ; \mathbb{R}\right) ; \\
& q(x) \geq 0, \quad \forall x \in \Omega ; \\
& q(x)=0, \quad \forall x \in \mathbb{R}^{3} \backslash \Omega .
\end{aligned}
$$

It is shown in Klibanov and Romanov [1] that problem (1) has a solution $u_{s}(x, k, y)$ in $C^{4}(|x-y| \geq \eta)$, for all $\eta>0$. The phaseless inverse scattering problem (PISP) [1-6] is to find the Schrödinger potential $q(x)$ if the information is given or partially given on the following types of scattering data measured in far-fields:

$$
\begin{aligned}
& \text { (PISP 1): } f(x, k, y):=\left|u_{s}(x, k, y)\right|^{2}, \\
& (x, k, y) \in S \times \mathbb{C} \times S ; \\
& \left(\text { PISP 2) }: g(x, k, y):=|u(x, k, y)|^{2},\right. \\
& \qquad(x, k, y) \in S \times \mathbb{C} \times S .
\end{aligned}
$$

We call the inverse problem given (5) the phaseless inverse scattering problem of type 1 and (6) type 2. This is a longstanding open problem in the inverse scattering theory of quantum mechanics [7-11], and the breakthrough is made in 
a series of papers by Klibanov and Romanov [1-6, 12]. The problem is typical in quantum mechanics and in the study of nano/microstructures when it is impractical to measure the phase of the scattered waves or the signals. The problem is also common in the inverse problems in micro/nanostructures, for example, electron microscopy, crystallography, medical imaging, and nanooptics. In crystallography, the phase information of a scattered wave field may be recovered if the intensity pattern at and between the Bragg peaks of diffracted wave is finely measured [13]. Additionally, due to the quality of many optical equipment and lens-like devices, the measurements may suffer from very serious optical aberration when the index of refraction is close to one. The engineering economy is that the phase retrieval algorithms may not be limited by the quality of the optical equipment. In astronomy, the research objects are usually distant stars, which are optically incoherent sources. In the cases of incoherent waves, the phase is stochastic. Hence, the optical signal is received/conceived as the intensity of the light, the square modulus of the complex-valued wave field, or the square modulus of the related Fourier transform. Moreover, the measurements usually are inflicted with very serious optical aberration caused either by atmospheric turbulence or by the imperfection of the optical imaging system. Thus, we ask if it is sufficient to reconstruct the scatterer/image source using only the intensity of the scattered wave fields or the signals.

Let us state two inverse uniqueness results in this paper.

Theorem 1. Let $f^{j}(x, k, y)$ be the square modulus of the complex-valued scattered wave field generated by Schrödinger potential $q^{j}, j=1,2$. If $f^{1}(x, k, y) \equiv f^{2}(x, k, y)$ for all $(x, k, y) \in S \times U \times S$ in a neighborhood $U$ intersected with real axis; then $q^{1} \equiv q^{2}$.

Theorem 2. Let $g^{j}(x, k, y)$ be the square modulus of the complex-valued total scattered wave field generated by potential $q^{j}, j=1,2$. If $g^{1}(x, k, y) \equiv g^{2}(x, k, y)$ for all $(x, k, y) \in$ $S \times U \times S$ in a neighborhood $U$ intersected with real axis; then $q^{1} \equiv q^{2}$.

Here we provide similar results to the ones in $[1,6]$. The neighborhood $U$ alternatively can be replaced by any accumulation point in the lower half complex plane or anywhere away from the poles of $u_{s}(x, k, y)$, and the source point is fixed on $S$. The advantage in this paper is that the argument does not rely on the Blaschke product of certain zero set.

Theorem 3. The total wave field $u^{j}(x, k, y)$ generated by potential $q^{j}$ each has at most a finite number of zeros for each fixed $(x, y)$. If $u^{1}(x, k, y)$ and $u^{2}(x, k, y)$ share the same zero set for each fixed $(x, y)$, say, $\left\{k_{1}(x), k_{2}(x), \ldots, k_{m(x)}(x)\right\}$, then $q_{1} \equiv q_{2}$.

For problem PISP 2, we will demonstrate later that the total wave $u(x, k, y)$ behaves asymptotically as an exponential function for large $k$ in the complex plane. We seek to apply the identity theorems in complex analysis to carry on the arguments. For PISP 1, the oscillation frequency of the scattered wave field is connected to the spectral invariant in the form of the Radon transform in analysis. In [1], it is shown that if $f(x, k, y)$ is provided for $k \gg 0$, the Schrödinger potential can be reconstructed from the inverse Radon transform of $\int_{L(x, y)} q(\xi) d \sigma$. In particular, the inverse existence and uniqueness on the potential $q$ are proved. In Theorem 1, we prove uniqueness by the information provided in a finite neighborhood of frequencies, and the existence follows accordingly. The proof of Theorem 1 is again the application of the spectral invariant $\int_{L(x, y)} q(\xi) d \sigma$.

In the last section, we discuss the inverse problem when only the phase information of the scattered wave fields is provided. It has been discussed in $[14,15]$.

In the real-world applications, the measured data is taken in real-valued frequencies. In Appendix, we discuss the inverse uniqueness when provided with only the measured data on the real axis. The mechanism is due to the Nevanlinna-Levin type of integral representation theorem.

\section{Preliminaries}

The fundamental element is the asymptotics $[1$, Theorem 1$]$

$$
\begin{array}{r}
u_{s}(x, k, y) \\
=\frac{i \exp \{-i k|x-y|\}}{8 \pi|x-y| k}\left\{\int_{L(x, y)} q(\xi) d \sigma+O\left(\frac{1}{k}\right)\right\}, \\
x \neq y, k \rightarrow \infty,
\end{array}
$$

in which $L(x, y)$ is the straight line connecting $x$ and $y$, and $d \sigma$ is the arc length. We extend the property into the lower half complex plane in the following lemma for the sake of a complex analysis [16].

Lemma 4 (Klibanov and Romanov). There exists some constant $C>0$ such that the following asymptotics holds in $\Im k<$ C

$$
\begin{array}{r}
u_{s}(x, k, y) \\
=\frac{i \exp \{-i k|x-y|\}}{8 \pi|x-y| k}\left\{\int_{L(x, y)} q(\xi) d \sigma+O\left(\frac{1}{k}\right)\right\}, \\
x \neq y .
\end{array}
$$

Proof. We review the fundamental solution of the hyperbolic equation $[1$, Sec. 3$]$

$$
\begin{aligned}
w_{t t} & =\Delta_{x} w-q(x) w+4 \pi \delta(x-y, t), \quad(x, t) \in \mathbb{R}^{4} ; \\
\left.w\right|_{t<0} & =0 .
\end{aligned}
$$

In particular,

$$
\begin{aligned}
w(x, t, y) & =w_{0}(x, t, y)+\widetilde{w}(x, t, y) H(t-|x-y|), \\
w_{0}(x, t, y) & =\frac{\delta(t-|x-y|)}{|x-y|},
\end{aligned}
$$


in which $H(t)$ is the Heaviside function and $\widetilde{w}(x, t, y)$ has the short-time behavior

$$
\lim _{t \rightarrow|x-y|^{+}} \widetilde{w}(x, t, y)=\frac{-1}{2|x-y|} \int_{L(x, y)} q(\xi) d \sigma
$$

Moreover, the solution of (9) satisfies the following properties. There exist constants $C_{1}>0$ and $C_{2}>0$ depending on $(y, q, R)$ such that

$$
\begin{aligned}
& \left|\partial_{t}^{k} w(x, t, y)\right| \leq C_{1} e^{-C_{2} t}, \\
& \quad \forall t \geq t_{0}, \quad \forall x \in\left\{x \in \mathbb{R}^{3}|| x-y \mid<R\right\}, k=0,1,2,
\end{aligned}
$$

for any $R>0$.

Let

$$
\begin{aligned}
V(x, k, y):=\frac{1}{4 \pi} \int_{0}^{\infty} w(x, t, y) e^{-i k t} d t, & \\
x & \neq y, k \in 0 i+\mathbb{R} .
\end{aligned}
$$

From the theory of $[1,5,17]$, we note that $u(x, k, y)=$ $V(x, k, y)$ is the solution of (1). Thus, we deduce from (1), (10), (12), (13), and (14) that

$$
\begin{aligned}
& u_{s}(x, k, y)=\frac{1}{4 \pi} \int_{|x-y|}^{\infty} \widetilde{w}(x, t, y) e^{-i k t} d t \\
& =-\frac{i \exp \{-i k|x-y|\}}{k}\left\{-\frac{1}{8 \pi|x-y|}\right. \\
& \left.\cdot \int_{L(x, y)} q(\xi) d \sigma-\frac{i}{4 \pi k} \partial_{t} \widetilde{w}\left(x,|x-y|^{+}, y\right)\right\} \\
& -\frac{1}{4 \pi k^{2}} \int_{|x-y|}^{\infty} \partial_{t}^{2} \widetilde{w}(x, t, y) e^{-i k t} d t,
\end{aligned}
$$

in which the last term could be of exponential growth for $\mathfrak{\Im} k \gg C_{2}>0$. For fixed $x \neq y$ and fixed $\mathfrak{\Im} k<C_{2}$, we deduce from the Riemann-Lebesgue lemma that

$$
\begin{array}{r}
\int_{|x-y|}^{\infty} \partial_{t}^{2} \widetilde{w}(x, t, y) e^{-i k t} d t \\
=\int_{|x-y|}^{\infty} e^{-i \Re k t}\left[\partial_{t}^{2} \widetilde{w}(x, t, y) e^{\Im k t}\right] d t=o(1), \\
\text { as } \mathfrak{R} k \longrightarrow \pm \infty .
\end{array}
$$

The lemma is thus proven.

For far-field behavior, we have

$$
u_{s}(x, k, y)=\frac{e^{i k|x|}}{|x|} u_{\infty}(\hat{x} ; y, k)+O\left(\frac{1}{|x|^{3 / 2}}\right),
$$

which holds uniformly for all $\hat{x}:=x /|x|, x \in \mathbb{R}^{3}$, and $u_{\infty}(\hat{x} ; y, k)$ is known as the scattering amplitude in the literature $[8,10,18,19]$. In this paper, we adopt the convention that $u_{\infty}(\hat{x} ; y, k)$ is defined analytically in $\Im k \leq 0$ and extended meromorphically from $\mathfrak{\Im} k \leq 0$ to $\mathbb{C}$. To avoid the poles of $u_{\infty}(\hat{x} ; y, k)$, we analyze the problem in $\Im k \leq 0$.
Lemma 5. The scattered wave field $u_{s}(x, k, y)$ in (17) is defined meromorphically in $\mathbb{C}$ with poles in $\mathfrak{\Im} k>0$, except for a finite number of purely imaginary $k$ 's that $k^{2}$ are the negative eigenvalues of (1). In particular, the poles of $u_{\infty}(\hat{x} ; y, k)$ are located as the mirror images of its zeros to the real axis.

Proof. This is well-known in scattering theory. Let us refer to [18-21] and in particular a few brief comments on the analytic structure of the scattering matrix in [20, Introduction]. There are only finitely many poles located in the lower half complex plane.

\section{A Proof of Theorem 1}

Let $u_{s}^{j}(x, k, y)$ be the scattered wave field induced by the Schrödinger potential $q^{j}(x)$, and

$$
f^{j}(x, k, y):=\left|u_{s}^{j}(x, k, y)\right|^{2} .
$$

We start with the assumption in Theorem 1; that is,

$$
f^{1}(x, k, y)=f^{2}(x, k, y), \quad(x, k, y) \in S \times U \times S .
$$

For each fixed $\mathfrak{\Im} k$ and $x \neq y, k \in U$, the function

$$
\begin{aligned}
f^{j}(x, k, y)= & \left\{\Re u_{s}^{j}(x, k, y)\right\}^{2}+\left\{\Im u_{s}^{j}(x, k, y)\right\}^{2} \\
= & \left\{\mathfrak{R} u_{s}^{j}(x, \mathfrak{R} k+i \Im k, y)\right\}^{2} \\
& +\left\{\mathfrak{I} u_{s}^{j}(x, \mathfrak{R} k+i \Im k, y)\right\}^{2}
\end{aligned}
$$

is real-analytic in $\mathfrak{R} k$ by avoiding the poles mentioned in Lemma 5. Hence, we extend the identity to a strip $H$ containing $0 i+\mathbb{R}$. We write

$$
H:=\left\{k \in \mathbb{C} \mid-\infty<\mathfrak{R} k<\infty,-\delta<\mathfrak{\Im} k<\delta<C_{2}\right\},
$$

in which the constant $C_{2}$ is specified in (13) and $\delta=\delta(U)>0$. Moreover, for each fixed $\mathfrak{R} k$ and $x \neq y, k \in H, f^{j}(x, \mathfrak{R} k+$ $i \mathfrak{} k, y)$ is analytic in $\mathfrak{\Im} k$. Thus, the identity (19) holds in

$$
\mathbb{C}^{-}:=\left\{k \in \mathbb{C} \mid-\infty<\mathfrak{R} k<\infty,-\infty<\mathfrak{\Im} k<C_{2}\right\},
$$

with possibly finite exception of poles. From (19) and (22), we deduce that

$$
\left|u_{s}^{1}(x, k, y)\right|=\left|u_{s}^{2}(x, k, y)\right|
$$

Accordingly, $u_{s}^{1}(x, k, y)$ and $u_{s}^{2}(x, k, y)$ have identical zero set in $\mathbb{C}^{-}$, and therein $u_{s}^{1}(x, k, y) / u_{s}^{2}(x, k, y)$ is analytic. Thus,

$$
\begin{aligned}
\ln \left\{\frac{u_{s}^{1}(x, k, y)}{u_{s}^{2}(x, k, y)}\right\}= & \ln \left|\frac{u_{s}^{1}(x, k, y)}{u_{s}^{2}(x, k, y)}\right| \\
& +i \arg \left\{\frac{u_{s}^{1}(x, k, y)}{u_{s}^{2}(x, k, y)}\right\} \\
= & i \arg \left\{\frac{u_{s}^{1}(x, k, y)}{u_{s}^{2}(x, k, y)}\right\},
\end{aligned}
$$


which is purely imaginary. From the Cauchy-Riemann equation in the theory of complex variable, we deduce that

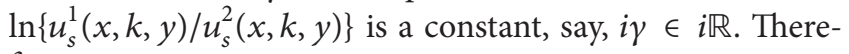
fore,

$$
\frac{u_{s}^{1}(x, k, y)}{u_{s}^{2}(x, k, y)}=e^{i \gamma}
$$

From Lemma 4, we consider

$$
\begin{aligned}
& \frac{u_{s}^{1}(x, k, y)}{u_{s}^{2}(x, k, y)} \\
& =\frac{(i \exp \{-i k|x-y|\} / 8 \pi|x-y| k)\left\{\int_{L(x, y)} q^{1}(\xi) d \sigma+O(1 / k)\right\}}{(i \exp \{-i k|x-y|\} / 8 \pi|x-y| k)\left\{\int_{L(x, y)} q^{2}(\xi) d \sigma+O(1 / k)\right\}} \\
& =\frac{\int_{L(x, y)} q^{1}(\xi) d \sigma+O(1 / k)}{\int_{L(x, y)} q^{2}(\xi) d \sigma+O(1 / k)} \geq 0, \quad k \in \mathbb{C}^{-} .
\end{aligned}
$$

We compare (25) and (26) for large $k$ to deduce that

$$
\int_{L(x, y)} q^{1}(\xi) d \sigma=\int_{L(x, y)} q^{2}(\xi) d \sigma
$$

for each fixed pair $(x, y)$ under the assumption in Theorem 1 .

For each line segment $L(x, y)$, we consider the inverse Radon transform $[1,4,5]$. For any number $a \in \mathbb{R}$, we consider the following sets:

$$
\begin{aligned}
& P_{a}:=\left\{x=\left(x_{1}, x_{2}, x_{3}\right) \in \mathbb{R}^{3} \mid x_{3}=a\right\} ; \\
& S_{a}:=S \cap P_{a},
\end{aligned}
$$

where $B_{a}=\sqrt{B^{2}-a^{2}}$ is the radius of the circle $S_{a},-B<a<$ $B$. Let us try to parametrize $L(x, y)$ in the setting of the Radon transform [22] on each $P_{a}$.

We consider the outer unit normal vector $n$ to the line $L(x, y)$ lying on the plane $P_{a}$. For each fixed $x_{3}=a$, we denote $\alpha \in(0,2 \pi]$ as the angle between normal vector $n$ and $x_{1}$-axis. Then $n=n(\alpha)=(\cos \alpha, \sin \alpha, 0)$. For the third coordinate, we set $s \in\left(-B_{a}, B_{a}\right)$ to be the signed distance of $L(x, y)$ to $(0,0, a)$. It is clear that there is one-to-one correspondence between pairs $(x, y)$ and $(n(\alpha), s)$. That is, we can identify $(x, y)$ and $(n(\alpha), s)$ by each other. Therefore, we can write for each fixed $a \in(-B, B)$

$$
L(x, y)=\left\{y_{a}:=\left(y_{1}, y_{2}, a\right) \in \mathbb{R}^{3} \mid y_{a} \cdot n(\alpha)=s\right\} .
$$

Thus, we derive the 2-dimensional Radon transform

$$
\int_{L(x, y)} q^{j}(\xi) d \sigma=\int_{y_{a} \cdot n(\alpha)=s} q^{j}\left(y_{a}\right) d \sigma, \quad j=1,2 .
$$

From (27) and the inverse uniqueness of the Radon transform [22], we deduce that $q^{1}\left(y_{a}\right) \equiv q^{2}\left(y_{a}\right)$ on each fixed $P_{a}$. Hence, we conclude that

$$
q^{1}(x) \equiv q^{2}(x)
$$

\section{A Proof of Theorem 2}

We start the total wave field $u^{j}(x, k, y)=u_{0}(x, k, y)+$ $u_{s}^{j}(x, k, y), j=1,2$. Therefore,

$$
\begin{aligned}
& u^{j}(x, k, y)=\frac{\exp \{-i k|x-y|\}}{4 \pi|x-y|} \\
& +\frac{i \exp \{-i k|x-y|\}}{8 \pi|x-y| k}\left[\int_{L(x, y)} q^{j}(\xi) d \sigma+O\left(\frac{1}{k}\right)\right] \\
& \quad=\frac{\exp \{-i k|x-y|\}}{4 \pi|x-y|}\left\{1+\frac{i}{2 k} \int_{L(x, y)} q^{j}(\xi) d \sigma\right. \\
& \left.+O\left(\frac{1}{k^{2}}\right)\right\}=\frac{\exp \{-i k|x-y|\}}{4 \pi|x-y|}\left\{1+O\left(\frac{1}{k}\right)\right\}, \\
& k \in \mathbb{C}^{-} .
\end{aligned}
$$

Hence,

$$
\begin{aligned}
\mid u^{j} & (x, k, y)-\frac{\exp \{-i k|x-y|\}}{4 \pi|x-y|} \mid \\
& <\left|\frac{\exp \{-i k|x-y|\}}{4 \pi|x-y|} O\left(\frac{1}{k}\right)\right| \\
& \leqslant\left|\frac{\exp \{-i k|x-y|\}}{4 \pi|x-y|}\right|,
\end{aligned}
$$

for $|k| \gg 0$ in $\mathbb{C}^{-}$. From Rouchés theorem in complex analysis, $u^{j}(x, k, y)$ and $\exp \{-i k|x-y|\} / 4 \pi|x-y|$ have identical number of zeros in regions away from the origin. Hence, $u^{j}(x, k, y)$ has only finitely many zeros near the origin. For $|k| \gg 0$, we apply the assumption in Theorem 2 to deduce that

$$
\begin{aligned}
\ln \left\{\frac{u^{1}(x, k, y)}{u^{2}(x, k, y)}\right\}= & \ln \left|\frac{u^{1}(x, k, y)}{u^{2}(x, k, y)}\right| \\
& +i \arg \left\{\frac{u^{1}(x, k, y)}{u^{2}(x, k, y)}\right\} \\
= & i \arg \left\{\frac{u^{1}(x, k, y)}{u^{2}(x, k, y)}\right\},
\end{aligned}
$$

which is again a purely imaginary function that extends to hold in $|k| \gg 0$ in $\mathbb{C}^{-}$. Hence, we conclude that $u^{1}(x, k, y) / u^{2}(x, k, y)=e^{i \delta}$ for some real constant $\delta$. Now we apply (32) to obtain

$$
e^{i \delta}=\frac{1+(i / 2 k) \int_{L(x, y)} q^{1}(\xi) d \sigma+O\left(1 / k^{2}\right)}{1+(i / 2 k) \int_{L(x, y)} q^{2}(\xi) d \sigma+O\left(1 / k^{2}\right)},
$$

$$
k \in \mathbb{C}^{-} \text {. }
$$

We deduce that $\delta=0$, and then $\int_{L(x, y)} q^{1}(\xi) d \sigma=$ $\int_{L(x, y)} q^{2}(\xi) d \sigma$. For each fixed line segment $L(x, y)$ and $P_{a}$, we apply the inverse Radon transform constructed in previous section and then prove $q^{1} \equiv q^{2}$. 


\section{A Proof of Theorem 3}

The total wave fields $u^{1}(x, k, y)$ and $u^{1}(x, k, y)$ have only finite number of zeros due to (32), from which we deduce that

$$
\begin{aligned}
\frac{u^{1}(x, k, y)}{u^{2}(x, k, y)} & =\frac{1+(i / 2 k) \int_{L(x, y)} q^{1}(\xi) d \sigma+O\left(1 / k^{2}\right)}{1+(i / 2 k) \int_{L(x, y)} q^{2}(\xi) d \sigma+O\left(1 / k^{2}\right)} \\
& =1+O\left(\frac{1}{k}\right), \quad k \in \mathbb{C}^{-} .
\end{aligned}
$$

For each fixed $(x, y)$, we denote

$$
P(k):=\left(k-k_{1}\right)\left(k-k_{2}\right) \cdots\left(k-k_{m}\right) .
$$

Accordingly, we consider the analytic function

$$
\begin{array}{r}
\frac{u^{1}(x, k, y)}{u^{2}(x, k, y)}=\frac{u^{1}(x, k, y) / P(k)}{u^{2}(x, k, y) / P(k)}=1+O\left(\frac{1}{k}\right), \\
k \in \mathbb{C}^{-} .
\end{array}
$$

We apply the Phragmén-Lindelöf Theorem [23-25] to conclude that $u^{1}(x, k, y) / u^{2}(x, k, y) \equiv 1$ in $\Im k \leq 0$. We repeat the argument in previous section to deduce that $\int_{L(x, y)} q^{1}(\xi) d \sigma=$ $\int_{L(x, y)} q^{2}(\xi) d \sigma$ for the fixed $L(x, y)$. The theorem is thus proven.

\section{Phase Only Problem}

The phase information of the scattered wave field or the Fourier transform plays a role in inverse problem $[14,15,26]$. In [15], two images are Fourier-transformed by swapping their phases which explains the importance of the phase information for image recovery.

Let us put the scattered wave fields in polar form

$$
\begin{aligned}
& u_{s}(x, k, y):=\left|u_{s}(x, k, y)\right| \exp \{i \arg \phi(x, k, y)\} \\
& u(x, k, y):=|u(x, k, y)| \exp \{i \arg \varphi(x, k, y)\}, \\
&(x, k, y) \in S \times \mathbb{C} \times S .
\end{aligned}
$$

In this section, we consider the inverse problems involving with phase information $\phi(x, k, y)$ and $\varphi(x, k, y)$.

Theorem 6. Let $\phi^{j}(x, k, y)$ be the phase information regarding the scatter wave field generated by $q^{j}, j=1,2$. If $\phi^{1}(x, k, y) \equiv$ $\phi^{2}(x, k, y)$ for all $(x, k, y) \in S \times U \times S$ in a neighborhood $U$ intersected with real axis, then $q^{1} \equiv e^{\gamma} q^{2}$, for some real constant $\gamma$.
Proof. We begin with the logarithmic function

$$
\begin{aligned}
\ln \left\{\frac{u_{s}^{1}(x, k, y)}{u_{s}^{2}(x, k, y)}\right\}= & \ln \left|\frac{u_{s}^{1}(x, k, y)}{u_{s}^{2}(x, k, y)}\right| \\
& +i \arg \left\{\frac{\phi^{1}(x, k, y)}{\phi^{2}(x, k, y)}\right\} \\
= & \ln \left|\frac{u_{s}^{1}(x, k, y)}{u_{s}^{2}(x, k, y)}\right| \\
& +i \arg \phi^{1}(x, k, y) \\
& -i \arg \phi^{2}(x, k, y) \\
= & \ln \left|\frac{u_{s}^{1}(x, k, y)}{u_{s}^{2}(x, k, y)}\right|,
\end{aligned}
$$

by theorem assumption. We note that it is purely real in $U$. Moreover, $\ln \left\{u_{s}^{1}(x, k, y) / u_{s}^{2}(x, k, y)\right\}$ is meromorphic in $\mathbb{C}$ by (17) if $u_{s}^{2}(x, k, y)$ is not identically zero. We deduce from the Cauchy-Riemann equation in the theory of complex variable that $\ln \left\{u_{s}^{1}(x, k, y) / u_{s}^{2}(x, k, y)\right\}$ is a real constant, say, $\gamma$ outside the possible poles in $\mathbb{C}$. Thus, we deduce that, outside some possible poles,

$$
\frac{u_{s}^{1}(x, k, y)}{u_{s}^{2}(x, k, y)}=e^{\gamma}
$$

Moreover, we apply Lemma 4 again to obtain

$$
\frac{u_{s}^{1}(x, k, y)}{u_{s}^{2}(x, k, y)}=\frac{\int_{L(x, y)} q^{1}(\xi) d \sigma+O(1 / k)}{\int_{L(x, y)} q^{2}(\xi) d \sigma+O(1 / k)}=e^{\gamma}
$$

$$
\mathfrak{J} k \leq 0 \text {. }
$$

Hence, for each $L(x, y)$,

$$
\begin{aligned}
\int_{L(x, y)} q^{1}(\xi) d \sigma & =e^{\gamma} \int_{L(x, y)} q^{2}(\xi) d \sigma \\
& =\int_{L(x, y)} e^{\gamma} q^{2}(\xi) d \sigma
\end{aligned}
$$

We repeat the inverse Radon transform argument in previous sections to conclude that $q^{1} \equiv e^{\gamma} q^{2}$.

In this case, the uniqueness is not available. We can carry out similar analysis to study total wave field $u(x, k, y)$.

Theorem 7. Let $\phi^{j}(x, k, y)$ be the phase information regarding the scattered total wave field generated by $q^{j}, j=1,2$. If $\phi^{1}(x, k, y) \equiv \phi^{2}(x, k, y)$ for all $(x, k, y) \in S \times U \times S$ in some neighborhood $U$ intersected with real axis, then $q^{1} \equiv q^{2}$. 
Proof. The proof is similar to Theorem 6. We repeat the proof of (40), (41), and (36) to deduce

$$
\begin{aligned}
e^{\gamma} & =\frac{u^{1}(x, k, y)}{u^{2}(x, k, y)} \\
& =\frac{1+(i / 2 k) \int_{L(x, y)} q^{1}(\xi) d \sigma+O\left(1 / k^{2}\right)}{1+(i / 2 k) \int_{L(x, y)} q^{2}(\xi) d \sigma+O\left(1 / k^{2}\right)} \\
& =1+O\left(\frac{1}{k}\right), \quad k \in \mathbb{C}^{-} .
\end{aligned}
$$

Here, we find $\gamma=0$. Then, $u^{1}(x, k, y)$ and $u^{2}(x, k, y)$ have the same zero set. It proves the theorem by repeating the argument in proving Theorem 3.

\section{Appendix}

\section{Complex Analysis and the Nevanlinna-Levin Theorem}

In the real-world applications, the scattered data are measured in real-valued frequencies. The assumption we have adopted so far is focused on the neighborhoods centered at real axis. In this appendix, we use complex analysis to relax the assumptions in Theorems 1, 2, 3, 6, and 7. The Fourier transforms (14) and (15) behave like exponential functions on many aspects. The integral representation theorem plays a role.

Definition A.1. Let $f(z)$ be an entire function. Let $M_{f}(r):=$ $\max _{|z|=r}|f(z)|$. An entire function of $f(z)$ is said to be a function of finite order if there exists a positive constant $k$ such that the inequality

$$
M_{f}(r)<e^{r^{k}}
$$

is valid for all sufficiently large values of $r$. The greatest lower bound of such numbers $k$ is called the order of the entire function $f(z)$. By the type $\sigma$ of an entire function $f(z)$ of order $\rho$, we mean the greatest lower bound of positive number $A$ for which asymptotically we have

$$
M_{f}(r)<e^{A r^{\rho}} .
$$

That is,

$$
\sigma:=\limsup _{r \rightarrow \infty} \frac{\ln M_{f}(r)}{r^{\rho}} .
$$

If $0<\sigma<\infty$, then we say $f(z)$ is of normal type or mean type.

Definition A.2. Let $f(z)$ be an integral function of finite order $\rho$ in the angle $\left[\theta_{1}, \theta_{2}\right]$. We call the following quantity as the indicator function of the function $f(z)$ :

$$
h_{f}(\theta):=\lim _{r \rightarrow \infty} \frac{\ln \left|f\left(r e^{i \theta}\right)\right|}{r^{\rho}}, \quad \theta_{1} \leq \theta \leq \theta_{2} .
$$

The type of a function is connected to the maximal value of the indicator function. We state it as the following lemma.

Lemma A.3 (Ja Levin [24, p. 72]). The maximum value of the indicator $h_{f}(\theta)$ of the function $f(z)$ on the interval $\alpha \leq \theta \leq \beta$ is equal to the type $\sigma$ of this function inside the angle $\alpha \leq \arg z \leq$ $\beta$.

Lemma A.4. The analytic function $u_{s}^{j}(x, k, y), j=1,2$, is bounded in the lower half complex plane.

Proof. We recall (8); that is,

$$
\begin{aligned}
& u_{s}(x, k, y) \\
& =\frac{i \exp \{-i k|x-y|\}}{8 \pi|x-y| k}\left\{\int_{L(x, y)} q(\xi) d \sigma+O\left(\frac{1}{k}\right)\right\}, \\
& x \neq y, \quad \mathfrak{s} k \leq 0 .
\end{aligned}
$$

Hence,

$$
\left|u_{s}(x, k, y)\right|=\exp \{\Im k|x-y|\} O\left(\frac{1}{k}\right), \quad \Im k \leq 0,
$$

which is bounded for negative $\Im k$.

We use the integral representation theorem due to Nevanlinna and Levin $[23,24,27]$.

Definition A.5. Let $f(z)$ be an analytic function in the upper half-plane. We say $u(z)$ is a harmonic majorant of $\log |f(z)|$ if $\log |f(z)| \leq u(z)$, and $u(z)$ is a harmonic function in the upper half-plane.

From Lemma A.4, the function $f(z)$ is bounded in the lower half-plane. Thus, $\log |f(z)|$ is bounded by a constant which is trivially harmonic.

Theorem A.6. Let $f(z)$ be an analytic function in $\{\Im z>$ $0\}$, and let the function $\log |f(z)|$ have a positive harmonic majorant in $\{\mathfrak{\Im} z>0\}$. Then

$$
\begin{aligned}
\log |f(z)|= & \sum_{k=1}^{\infty} \log \left|\frac{z-a_{k}}{z-\bar{a}_{k}}\right|+\frac{y}{\pi} \int_{-\infty}^{\infty} \frac{\log |f(t)|}{|t-z|^{2}} d t \\
& +\sigma y
\end{aligned}
$$

where $\left\{a_{k}\right\}$ are the zeros of $f(z)$ in $\{\Im z>0\}$.

Proof. We refer the proof to [27, p. 104] and the remark therein. We also refer more connection of the integral representation theorem to the other analytic properties of the function [27, p. 115, p. 116].

Theorem A.7. If $f^{1}(x, k, y)=f^{2}(x, k, y)$ for $k$ in a neighborhood $I \subset \mathbb{R}+0 i$ with $(x, y)$ fixed, then $\left|u_{s}^{1}(x, k, y)\right|=$ $\left|u_{s}^{2}(x, k, y)\right|$ holds in $\mathbb{C}^{-}$. 
Proof. Given $\left|u_{s}^{1}(x, k, y)\right|^{2}=\left|u_{s}^{2}(x, k, y)\right|^{2}$ for all $k \in I$, the identity for the real-analytic functions extends to hold in real axis. Hence, we have

$$
\left|u_{s}^{1}(x, k, y)\right|=\left|u_{s}^{2}(x, k, y)\right|, \quad k \in \mathbb{R}+0 i .
$$

For fixed $(x, y)$, the type of $u_{s}^{j}(x, k, y), j=1,2$, is zero in $\Im k \leq$ 0 by following Definitions A.1 and A.2, Lemmas A.3 and A.4. In (A.7), let us set $\sigma=1$. Therefore, we apply Theorem A.6 in $\Im k \leq 0$ to deduce

$$
\begin{aligned}
& \log \left|u_{s}^{1}(x, k, y)\right|-\log \left|u_{s}^{2}(x, k, y)\right| \\
& =\sum_{n=1}^{m} \log \left|\frac{k-a_{n}}{k-\bar{a}_{n}}\right|-\sum_{n=1}^{m^{\prime}} \log \left|\frac{k-a_{n}^{\prime}}{k-\bar{a}_{n}^{\prime}}\right|, \quad \Im k \leq 0,
\end{aligned}
$$

where $\left\{a_{n}\right\}$ and $\left\{a_{n}^{\prime}\right\}$ are the zeros of $u_{s}^{1}(x, k, y)$ and $u_{s}^{2}(x, k, y)$, respectively, in the lower half-plane. That is,

$$
\frac{\left|u_{s}^{1}(x, k, y)\right|}{\left|u_{s}^{2}(x, k, y)\right|}=\frac{\prod_{n=1}^{m}\left|\left(k-a_{n}\right) /\left(k-\bar{a}_{n}\right)\right|}{\prod_{n=1}^{m^{\prime}}\left|\left(k-a_{n}^{\prime}\right) /\left(k-\bar{a}_{n}^{\prime}\right)\right|},
$$

$$
\mathfrak{I} k \leq 0
$$

which is the absolute value of a rational function in $k$.

From (A.8) and (A.10), we deduce that

$$
\frac{\left|u_{s}^{1}(x, k, y)\right|}{\left|u_{s}^{2}(x, k, y)\right|} \equiv 1 \text {. }
$$

Moreover, the identity extends to be valid up to $\mathfrak{s}<<C_{2}$ discussed as in (22). The theorem is thus proven.

\section{Competing Interests}

The author declares that there is no conflict of interest regarding the publication of this manuscript.

\section{Acknowledgments}

This author's work is supported by Ministry of Science and Technology, NSC 99-2115-M-194-004.

\section{References}

[1] M. V. Klibanov and V. G. Romanov, "Reconstruction formula for a 3-d phaseless inverse scattering problem for the Schrödinger equation," https://arxiv.org/abs/1412.8210.

[2] M. V. Klibanov and V. G. Romanov, "Explicit formula for the solution of the phaseless inverse scattering problem of imaging of nano structures," Journal of Inverse and Ill-Posed Problems, vol. 23, no. 2, pp. 187-193, 2015.

[3] M. V. Klibanov and V. G. Romanov, "The first solution of a long standing problem: reconstruction formula for a 3$\mathrm{d}$ phaseless inverse scattering problem for the Schrödinger equation," Journal of Inverse and Ill-Posed Problems, vol. 23, no. 4, pp. 415-428, 2015.
[4] M. V. Klibanov and V. G. Romanov, "Two reconstruction procedures for a $3 \mathrm{D}$ phaseless inverse scattering problem for the generalized Helmholtz equation," Inverse Problems, vol. 32, no. 1, Article ID 015005, 2016.

[5] M. V. Klibanov and V. G. Romanov, "Reconstruction procedures for two inverse scattering problems without the phase information," SIAM Journal on Applied Mathematics, vol. 76, no. 1, pp. 178-196, 2016.

[6] M. V. Klibanov, "Phaseless inverse scattering problems in three dimensions," SIAM Journal on Applied Mathematics, vol. 74, no. 2, pp. 392-410, 2014.

[7] K. Chadan and P. C. Sabatier, Inverse Problems in Quantum Scattering Theory, Springer, New York, NY, USA, 1977.

[8] D. Colton and R. Kress, Inverse Acoustic and Electromagnetic Scattering Theory, vol. 93 of Applied Mathematical Sciences, Springer, New York, NY, USA, 3rd edition, 2013.

[9] N. Hurt, Phase Retrieval and Zero Crossings: Mathematical Methods in Image Construction, Kluwer Academic Publishers, Boston, Mass, USA, 1989.

[10] V. Isakov, Inverse Problems for Partial Differential Equations, vol. 127 of Applied Mathematical Sciences, Springer, New York, NY, USA, 1998.

[11] R. G. Newton, Inverse Schrödinger Scattering in Three Dimensions, Springer, New York, NY, USA, 1989.

[12] M. V. Klibanov, "Uniqueness of two phaseless nonoverdetermined inverse acoustics problems in 3-d," Applicable Analysis, vol. 93, no. 6, pp. 1135-1149, 2014.

[13] D. Sayre, "Some implications of a theorem due to Shannon," Acta Crystallographica, vol. 5, no. 6, p. 843, 1952.

[14] M. V. Klibanov, "The definition of a finite function by its Fourier transform argument," Doklady Akademii Nauk SSSR, vol. 289, no. 3, pp. 539-540, 1986.

[15] Y. Shechtman, Y. C. Eldar, O. Cohen, H. N. Chapman, J. Miao, and M. Segev, "Phase retrieval with application to optical imaging: a contemporary overview," IEEE Signal Processing Magazine, vol. 32, no. 3, pp. 87-109, 2015.

[16] L.-H. Chen, "An inverse uniqueness of a phaseless scattering problem by zero-crossings," https://arxiv.org/abs/1605.05257.

[17] B. R. Vainberg, Asymptotic Methods in Equations of Mathematical Physics, Gordon \& Breach Science, New York, NY, USA, 1989.

[18] P. D. Lax and R. S. Phillips, Scattering Theory, Academic Press, Boston, Mass, USA, 1989.

[19] R. B. Melrose, Geometric Scattering Theory, Cambridge University Press, New York, NY, USA, 1995.

[20] A. Sá Barreto, "Remarks on the distribution of resonances in odd dimensional Euclidean scattering," Asymptotic Analysis, vol. 27, no. 2, pp. 161-170, 2001.

[21] J. Sjöstrand and M. Zworski, "Lower bounds on the number of scattering poles II," Journal of Functional Analysis, vol. 123, no. 2, pp. 336-367, 1994.

[22] F. Natterer, The Mathematics of Computerized Tomography, John Wiley \& Sons, Chichester, UK, 1986.

[23] R. P. Boas, Entire Functions, Academic Press, New York, NY, USA, 1954.

[24] B. Ja Levin, Distribution of Zeros of Entire Functions, Translations of Mathematical Monographs, American Mathematical Society, 1972.

[25] E. C. Titchmarsh, The Theory of Functions, Oxford University Press, New York, NY, USA, 2nd edition, 1939. 
[26] M. V. Klibanov, P. E. Sacks, and A. V. Tikhonravov, “The phase retrieval problem," Inverse Problems, vol. 11, no. 1, pp. 1-28, 1995.

[27] B. Ya. Levin, Lectures on Entire Functions (Translations of Mathematical Monographs), vol. 150, American Mathematical Society, Providence, RI, USA, 1996. 


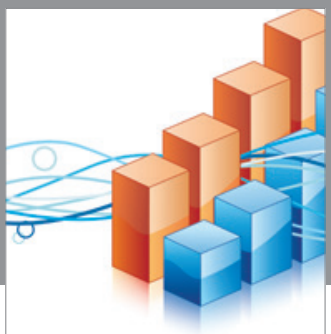

Advances in

Operations Research

vatem alat4

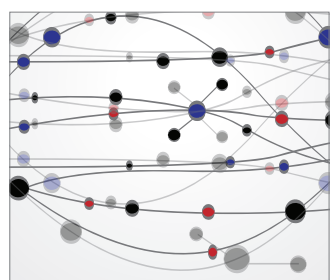

\section{The Scientific} World Journal
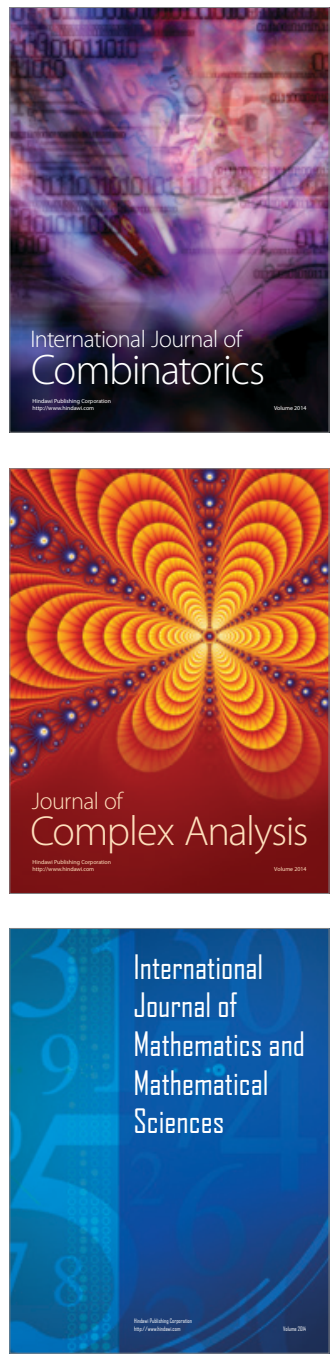
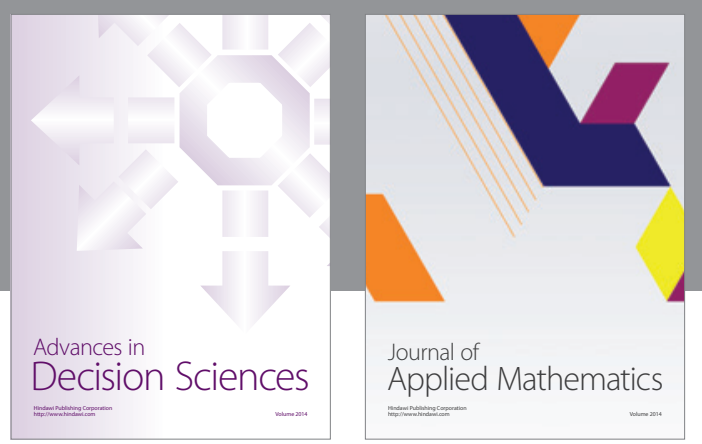

Algebra

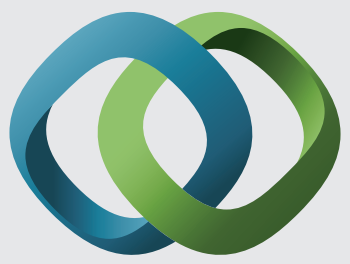

\section{Hindawi}

Submit your manuscripts at

http://www.hindawi.com
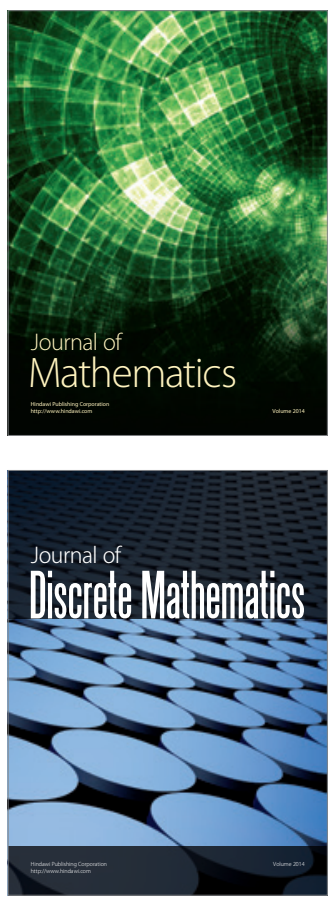

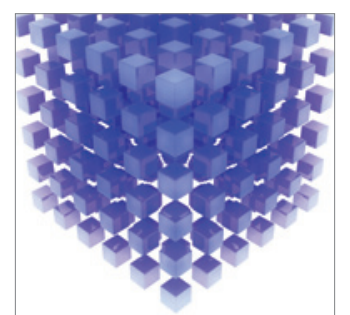

Mathematical Problems in Engineering
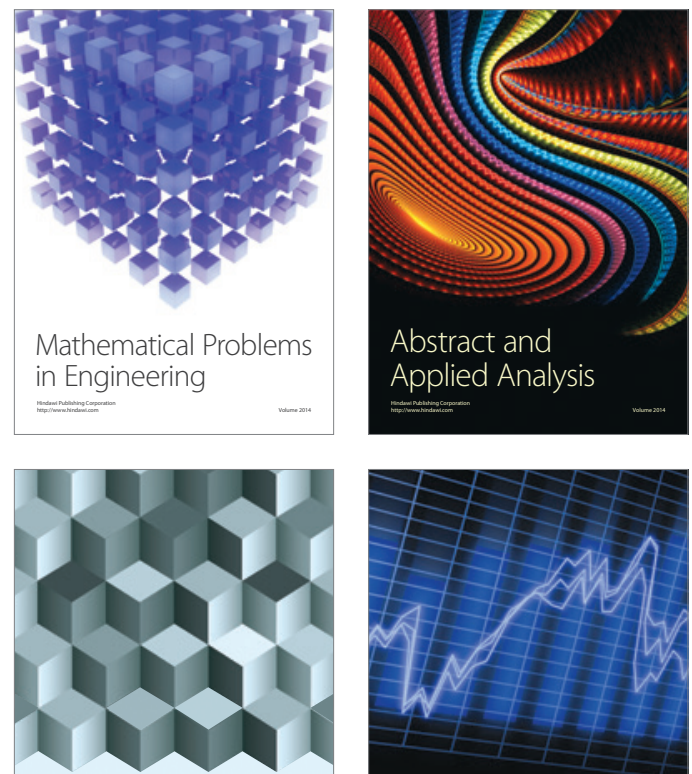

Journal of

Function Spaces

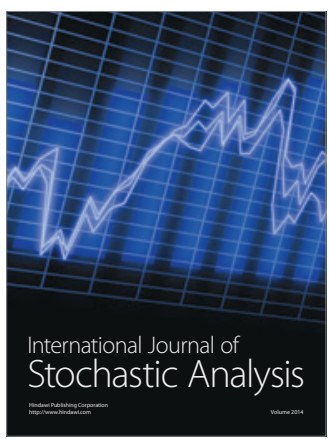

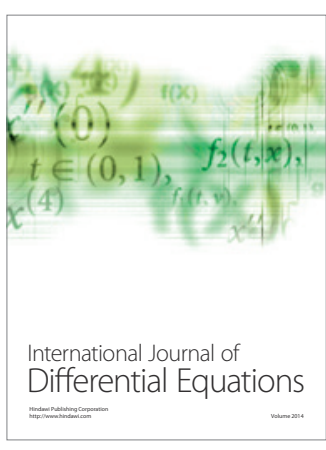
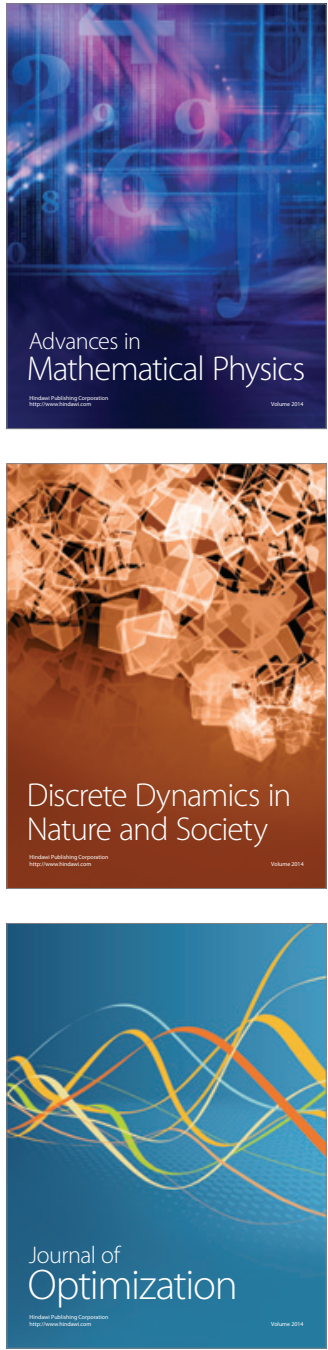